\title{
Development of an Excel-Based Software Solution to Collegiate EMS Shift Scheduling \& Placement
}

\author{
David Gordon, EMT; Ezra Brooks, EMT; Donavon Sandoval, EMT; Kaitlyn Boyle, EMT
}

POSTER PRESENTATION ABSTRACT | PROGRAM DEVELOPMENT \& EVALUATION CATEGORY

Introduction: For variably-scheduled volunteer EMS organizations, scheduling becomes a priority to sustain services to the community. EMS leadership must fill each shift by placing EMTs on shifts that best match their availability. With approximately 50 EMTs, 20 of whom lead calls, the University of Pennsylvania's Medical Emergency Response Team (MERT) covers 18 shifts per week, with 2-4 EMTs on each shift. Each week, MERT volunteers submit availability digitally. Prior to implementation of the current system, the scheduling coordinator would aggregate the schedule options submitted and assemble the schedule by hand. This system was inefficient and imperfect.

Program Development \& Implementation: While there are commercial solutions available, over Fall 2018, MERT developed an Excel-based solution customized to the agency's needs. The result is an Excel-spreadsheet-based algorithm that interprets the availability and optimizes the number of covered shifts based off availability responses. A binary variable was generated for each person and each potential shift. Constraints limited total shifts an EMT could take, defined a filled shift, and ensured that EMTs were only slated when they were available. This generated a linear model that the Excel OpenSolver add-on optimizes to preferentially fill shifts and match providers to empty shifts. The solved model is then interpreted by an array function with conditional formatting to output a formatted schedule. Additional options are available on the front-end to force the solver to provide for shifts with two crews, athletics events, and/or call reviews.

Program Evaluation: With implementation over 8 scheduling cycles, the model increased the proportion of fully covered shifts from $56 \%$ to $76 \%(P<0.03)$ and partial coverage from $85 \%$ to $97 \%$ when the schedule was released $(P<0.05)$. This model now allows maximal coverage when the schedule is first released and has dramatically reduced the time needed to manually schedule providers.

Discussion/Conclusions: A similar model could potentially be utilized by other collegiate EMS organizations, without cost, to optimize availability and maximize coverage.

Author Affiliations: Medical Emergency Response Team, University of Pennsylvania, Philadelphia, PA, USA

Address for Correspondence: David Gordon, EMT

E-mail: davgor@sas.upenn.edu

Conflicts of Interest/Funding Sources: By the JCEMS Submission Declaration Form, all authors are required to disclose all potential conflicts of interest and funding sources. The authors declared that they have no conflicts of interest. The authors declared that they received funding from University of Pennsylvania Division of Public Safety, University of Pennsylvania Division of the Vice Provost for University Life, and University of Pennsylvania Robert A. Fox Leadership Program for the program and/or research described in this abstract.

Ethical Compliance: The authors attest that the research associated with this abstract was conducted in accordance with the JCEMS Ethics Guidelines.

Submission History: Received January 6, 2019; accepted for presentation and publication February 11, 2019.

Poster Presentation: This abstract was presented as a poster at the Academic Poster Session of the $26^{\text {th }}$ Annual Conference of the National Collegiate Emergency Medical Services Foundation; February 23, 2019; Pittsburgh, PA, USA. The authors received the First Place Award for the Best Poster Presentation Competition.

Published Online: December 31, 2019
Published in Print: December 31, 2019 (Volume 2: Supplemental 1)

Reviewer Information: In accordance with JCEMS editorial policy, poster presentation abstracts undergo double-blind peer-review by at least two reviewers (JCEMS Editorial Board members and/or independent reviewers) prior to acceptance for presentation and publication. JCEMS thanks the anonymous reviewers who contributed to the review of this work.

Copyright: (C) 2019 Gordon, Brooks, Sandoval \& Boyle. This is an open access abstract distributed under the terms of the Creative Commons Attribution 4.0 International (CC BY 4.0) License, which permits unrestricted use, distribution, and reproduction in any medium, provided the original author and source are credited. The full license is available at: https://creativecommons.org/licenses/by/4.0/ Electronic Link: https://doi.org/10.30542/JCEMS.2019.02.S1.01 